\title{
IWARN: A people-centered approach for early warning
}

\author{
Paloma Díaz, Mónica Sánchez-Francisco, Teresa Onorati; Alvaro Montero; Ignacio Aedo \\ Computer Science and Engineering Department, Universidad Carlos III de Madrid \\ pdp@inf.uc3m.es
}

\begin{abstract}
Early warning is the activity of the mitigation phase concerned with monitoring precursors of a potential hazard to decide whether it is evolving to real risk and eventually initiate an early response. The first step consists of collecting updated and reliable data to support situational awareness from emergency operators. Data-centered Early Warning Systems (EWS) are focused on gathering data and run simulations to support decision-makers. A more sustainable approach consists of a people-centered EWS that takes profit from citizens who act as intelligent sensors collecting and sharing purposeful information. This people-centered approach can contribute to raising community awareness of the local environment and its vulnerabilities. In this paper, we introduce iWarn, a system relying upon mobile computing and gamification to integrate citizens in this process. The system has been developed following an action research approach to involve different stakeholders, including professionals, volunteers, and citizens.
\end{abstract}

\section{Introduction}

The response to an emergency starts before anything has happened during the preparedness and mitigation phases when communities need to identify the potential hazards they are exposed to and get ready to react as efficiently as possible. Early warning is part of the mitigation phase. It consists of monitoring precursors of a potential hazard to decide whether it is evolving to a real risk so that an early response can be fired [1]. For instance, wildfire early warning implies checking precursors like vegetation dryness and weather to help emergency operators decide when the situation is risky enough to issue an alert [2]. Early Warning ICTbased Systems (EWS henceforth) have been developed to monitor wildfires, flooding, earthquakes, or tsunamis, relying mainly on collecting data from sensors and using simulations of the event evolution $[3,4,5]$. Such EWS are mainly data-centered since they focus on collecting and managing data. Other social and cultural issues could provide valuable information to understand the potential impact of a specific hazard in a community. For instance, in the wildfire scenario, it would be helpful also to check things like the existence of fuels in the area, uncontrolled human activities (e.g., spontaneous gatherings or seasonal settlements) [3], groups of citizens with special needs, community habits and available resources to mobilize if required. In [1], the authors introduce a decentralized and people-centered EWS to generate awareness about vulnerabilities and better prepare for hazards the affected communities. The EWS is based on networks of people who share information mainly through radio. This approach was followed by communities of practitioners like FEWSN, AEWN, REMER, or RACES [6].

Most of these people-centered EWS rely upon communities of volunteers, whilst the goal of early warning "is to empower individuals and communities, threatened by hazards and crisis situations, to act in an appropriate manner so as to reduce the possibility of personal injuries and material damages" [7]. In this paper, we posit that to involve citizens' needs in the process, emergency management (EM henceforth) has to turn into a coproduction service that relies upon and strengthens communities whilst coping with the requirements and protocols of the EM organizations [8]. A coproduction of EWS based on the fruitful participation and collaboration of experts, volunteers, and citizens will be more efficient and sustainable. It will be more efficient since varied resources, abilities, and capabilities can be integrated to get a more complete picture of the situation and, therefore, improve situation awareness [9]. It will be more sustainable as coproduction relies upon the social capital of communities [10] and their digital social capital [11], that is, online communities that pursue a common goal.

In this paper, we introduce iWarn, a peoplecentered EWS relying upon mobile computing to integrate citizens in this process that has been developed following an action research approach. The design of the system involved professional EM workers and volunteers in an iterative cycle of analysis, action, assessment, and learning where under-designed prototypes and models helped to focus attention on how 
to empower participants and decision-makers. In the last cycle the resulting prototype was validated with citizens.

The remaining of the paper is organized as follows. Section 2 reviews related works on people centered EM that motivate this research. Section 3 introduces the iWarn system whose design process following an action research approach is described in section 4. Section 5 includes the last evaluation and finally, section 6 draws some conclusions.

\section{People-centered early warning}

After the devastating effects of 2004 Indian Ocean tsunamis, early warning emerged as a key activity to decrease the impact of natural or man-made hazards by supporting better situation awareness and early response protocols [1]. Early warning is often implemented as an iteration of collecting data and monitoring precursors, analyzing data to identify hazards, communicating hazards, and starting early response activities to minimize the hazard impact [1]. There are already EWS that make use of sensors and radar based information as well as simulations of the event evolution $[3,4,5]$. What we propose in this work is to integrate citizens in this process as claimed in $[1,7,8,12]$, in order to provide a better service to the community and to build more resilient communities, aware of the risks they might be exposed to and with capacity to recover effectively from disasters [13]. To the best of our knowledge there are no similar systems to support participatory EWS.

Local-level or participatory EM roots on the idea that "emergency management capacity is built from the ground up" [14]. By promoting the participation of citizens as active agents [15] we can contribute to generate resilient communities that have their own capacities and resources to deal with hazards and disasters [10]. Whilst in a command-and-control system early warning will depend on data-centered approaches managed by governmental agencies a participatory perspective will follow a people-centered approach where citizens contribute as intelligent sensors [12]. In this case, citizens are not only reactive to the crisis, but they can also be proactive and be prepared to be less vulnerable. People-centered EWS require empowering both citizens but also organizations that hold the EM responsibility. Citizen-generated content can only be useful if properly aggregated, filtered and reorganized to generate knowledge that can be understood and managed by EM workers.

Mobile computing technologies provide the means to collect different types of data about potential hazards and their evolution, using both artificial and human sensors. For instance, weSenseIt combines human and artificial sensors for flood management [12]. In this paper we explore interaction design issues related with people-centered EWS and, in particular, we aim at understanding how technological tools have to be designed to substantively empower both citizens and institutions to act in an integrated way. For that to be possible, EM workers and decisions makers must have a stake in the design process to decide which functionalities should be included to guarantee that the final outcomes will be useful for them and will not interfere with their protocols. On the other side, citizens need to be engaged in a process whose utility is not selfevident. Early warning is not an intrinsically rewarding or motivating activity for citizens insofar the risk is not perceived yet. For that reason, we will explore gamification as a strategy to increase system acceptance. Gamification is broadly defined as the use of game-elements in non-game context [15]. In the EM context gamification has been applied to increase motivation with training purposes in $[17,18]$.

\section{The iWarn EWS}

People-centered EWS aims at preparing operators, volunteers, and citizens to be ready when an emergency occurs so its impact on the community can be reduced. The participation of citizens and volunteers is crucial to help the operators in collecting information about a specific situation by continuously checking the evolution the hazard precursors and the status of the potentially affected area. In the EM operation center, this information can be used to understand how the hazard is evolving and determine whether an alert or warning to the population must be issued. In this context, iWarn is an EWS supporting professionals, volunteers, and citizens in monitoring potential risks in a specific geographical area. The design of iWarn is based on the results collected during the two iterations of the action research cycle described in section 4. For the sake of clarity, the description of the final system is included before the method.

\section{1. iWarn Architecture}

The iWarn architecture consists of two main components (see Figure 1): the operation desktop and the mobile application. The desktop system is where early warning monitoring is carried out. Information from precursors of potential risks can be received from external sources or directly added by emergency operators. With this information the operator decides whether to create or not a mission to collect information. The mission is the gamified concept introduced to motivate citizens to participate and it is performed in the mobile application. Citizens can enroll missions by invitation, and they join them as part of a collaborative 
team. Potential participants receive notifications from the system to join the mission and if they accept they can start sending back information. Operators also assign a leader to each team who will be in charge of monitoring and orchestrating participation. The leader was included to alleviate the operator's tasks since she will filter the information collected and she will also guide participants. The leader can be an operator or a volunteer with extensive experience in managing critical situations. This approach follows the participatory structure defined in [8] where citizens are divided into sensors and trusted sensors, nodes and agents depending on their known capability to contribute to a mission.

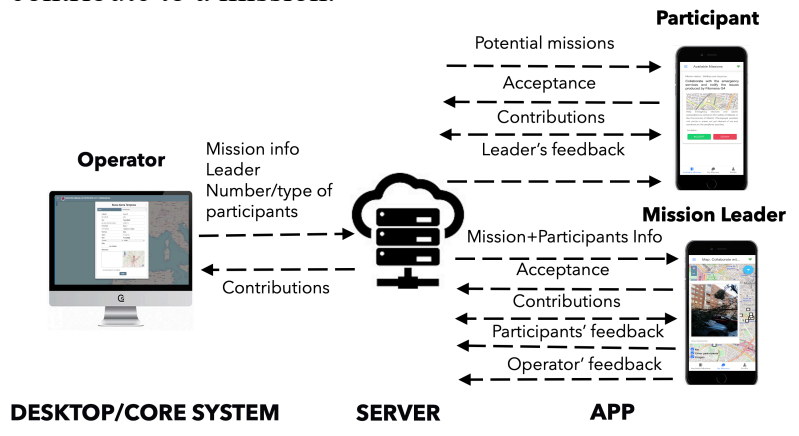

Figure 1. The iWARN Architecture

\subsection{The iWarn Desktop}

iWarn desktop allows EM operators to manage geolocalized information about early warnings and integrate citizen participation. To support usage in a multidevice environment, it is designed as a responsive website (see screenshots in Figure 2).

Considering that the use of geographic data is critical for any emergency situation, the map is the main element of the interface (see Figure 2a). Active early warnings are visualized as colored areas, so that the operator has visual feedback on open operations. The color represents the severity of the early warning for which the official scale defined by the Spanish Civil Protection is used. Users can interact with each shape on the map to get more details as well as create a new warning, receive new notifications and access to their profile.

New early warning operations are created by using a form whose fields follow the official templates used by the Spanish Civil Protection (Figure 2b). Early warnings can also be imported from other sources. The system automatically checks the news issued by the National Weather Agency and allows the operator to import them. In the previous version early warning operations were directly linked to citizens missions so they were created at the same time. Considering the results of the focus group (see below) this action was detached from the mission, so that the operator can decide whether an early warning requires citizen participation or not and still use the same interface to check all open operations.

When creating a mission (Figure 2c), operators have to specify several data including: the title, the leader, the communication channel (i.e., mobile app, messaging services, social networks, and radio), the profile of participants (e.g., REMER, volunteers, or citizens), the quantity of information to collect (i.e., the maximum number of participants and the maximum number of images to send), the gamified reward in case of successful participation.

Operators can check the status of open early warnings (see Figure 2d) by clicking on the shapes of the map. This action opens a popup containing more details about the warning: the author to promote credibility, the affected area, a description, and three option to archive the warning if solved, open a direct communication channel with the author and navigate the multimedia content sent by the citizens.

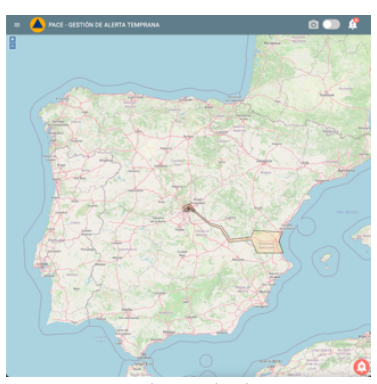

a. Map based view

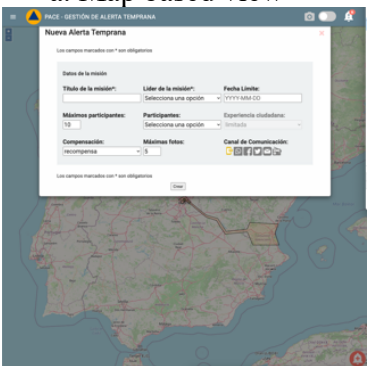

c. Creating missions

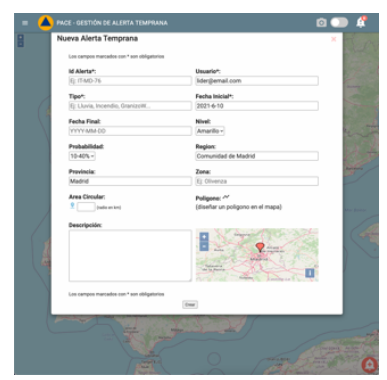

b. Creating early warnings

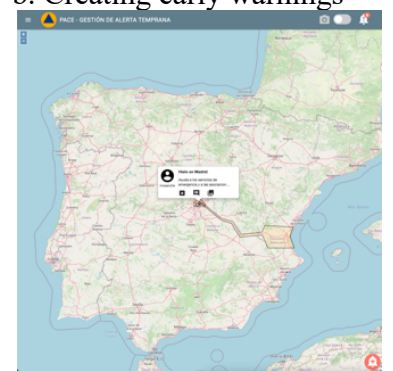

d. Checking results
Figure 2. Interface of the iWarn desktop system 


\section{3. iWarn Mobile Application}

The mobile application is used by leaders and participants to collect pictures on the affected areas and precursors of a potential hazard. Leaders are in charge of coordinating the citizens' activities and communicating with the central system. The app is developed using Ionic Framework 5 and Angular and compiled for Android and iOS platforms.

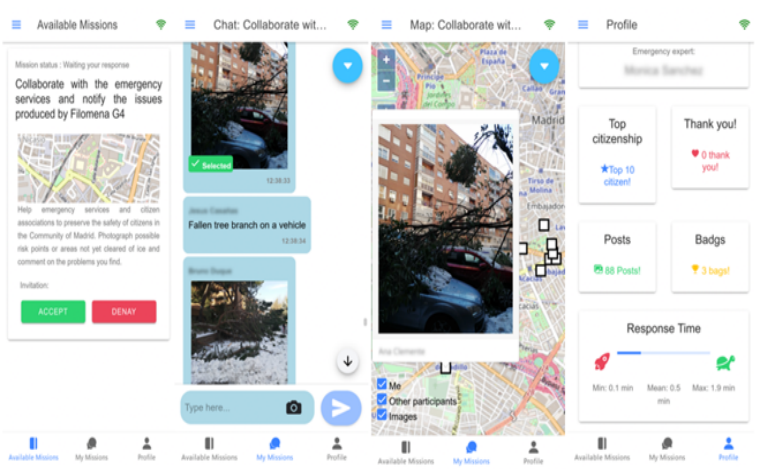

Figure 3. Screenshots of iWarn mobile app. From left to the right: available missions, chat, map, and profile.

After logging in, users can access three sections: Available Missions, My Missions, and Profile (see Figure 3). The first section shows the list of invitations received (see Figure 3 available missions screen). Leaders and volunteers can accept or not participating in a mission, but they cannot negotiate the terms of the mission. Once leaders accept a mission, they can send invitations to potential participants. The section $M y$ Missions shows the ongoing and completed missions. The ongoing missions are associated with a chat where the leader and the volunteers share geolocated images and text messages. The leader is responsible for moderating the chat to guide the volunteers, provide further information, and filter the most valuable content for the operator (see Figure 3, chat screen). It is also possible to visualize the images and the current location of the active participants to know which areas have already been explored (see Figure 3 map screen). The leader also has a gallery to select the most valuable images, a participant list to invite more volunteers and give them a personal thank you message, and a report section to close the mission and notify the central system. The last section of the application is the user Profile with information about the activity measured as the number of sent messages, completed missions, and received thank you (see figure 3 profile screen).

\section{Action research cycle}

The work of our research group on EWS started by a petition of the Spanish General Directorate of Civil
Protection and Emergencies (DGPCE) to provide an ICT platform for the existing radio amateurs network (REMER) whose members participate in early warning activities. The initial goal was to incorporate the benefits of mobile computing as an additional communication channel to the radio, for which the remerWEB platform was deployed [19]. The interface and functionalities were codesigned with members of DGPCE in charge of the REMER network and was evaluated with REMER members $[6,19]$. The next step was to open participation to citizens so that the EWS could be used to improve community's awareness and preparation. From a technical point of view incorporating a different kind of user might not seem a challenge, but from a point of view of technology acceptance is a design wicked problem. As discussed in the previous sections, integrating citizens in EM activities implies understanding why and how they will participate without interfering with existing protocols nor putting themselves in danger. We carried out different studies to understand how to proceed as reported in [8] and finally decided to apply an action research approach [20]. The design of the system involved professional EM workers and volunteers in an iterative cycle of analysis, action, assessment and learning. Prototypes and models helped to identify how iWarn could empower both participants and decision makers.

\subsection{First Iteration: defining the iWarn Architecture}

The first iteration of the action research approach started by studying not only the literature about EWS and related concepts like coproduction and communitybased EM (see section 2), but also the domain of application, to identify opportunities to integrate a technological EWS. With that purpose, two exploratory focus groups were run with professional EM workers and volunteers who explored design artifacts like models and under-designed prototypes with a view to elicit the system requirements $[8,21,22]$. Since operational technologies are challenging to deploy in the EM domain due to political and organizational issues, we focused on analyzing prototypes and models to provide valuable hints on integrating acceptable technology in real situations.

The main objective of iWarn is to offer a platform to ease the flow of information among EM agencies, other official sources like the weather or traffic monitoring services, volunteers, and citizens. The potential usage of this kind of tool in a real scenario depends mainly on whether EM workers rely on collected data and are willing to use them in their daily duties. To understand how EM workers perceive the designed platform, we run an expert focus group as 
reported in [22]. After this focus group, several improvements were identified and implemented as described in [21]. The main result of this iteration was the architecture described in section 3.1 and figure 1.

This architecture that considered the needs of EM workers was evaluated in a second round using an under-designed prototype that showed the functionalities though it was not fully implemented as described below.

\subsection{Second Iteration: Asynchronous Expert Focus Group}

In the second iteration we collected feedback through an expert focus group (EFG) with several volunteers who evaluated the prototype resulting from the previous cycle [22]. It was crucial to involve people with knowledge on what early warning is and how it impacts EM, so we invited five members of the REMER network with extensive experience and reputation. Due to the participants' schedules and geographical locations, which made it impossible to run synchronous and on-site activities, we organized an online asynchronous EFG. We followed an approach called bulletin board, where participants use an online tool to discuss a set of topics during a limited number of days [20]. In our case, the bulletin board was a multimedia blog with ten topics related to different aspects of iWarn (see Table 1). The topics selected aimed at answering 3 questions on iWarn utility: (Q1) Which are the main advantages and limitations of the system?; (Q2) Which real scenarios could take advantage of the platform?; and (Q3) Is gamification useful for improving the information flow between official channels and citizens?. Though this approach can have limitations in terms of interaction between remote participants its main benefits are convenience and flexibility to adapt to the participants availability. Moreover, participants do not have the pressure of other people waiting for their answers, like it happens during a face-to-face or synchronous activity [23], and they have more time to think over their opinions and suggestions. We used videos since to test the system you have to set up a full team with operators, leaders and participants and the goal of this EFG was to validate the design decisions before running a full experiment.

Table 1. The ten topics used in the focus group with the associated questions (Q1, Q2, Q3).

\begin{tabular}{cl}
\hline Id & Topics \\
\hline 1 & $\begin{array}{l}\text { Overall opinions and suggestions about iWarn } \\
\text { (Q1) }\end{array}$ \\
2 & $\begin{array}{l}\text { Configuration/notification of early warnings } \\
\text { (Q1) }\end{array}$ \\
\hline
\end{tabular}

3 Receiving and sending information using the iWarn mobile app (Q1)

4 Functionalities of the iWarn mobile app (Q1)

5 Participation in a mission (Q1)

6 Participation in a training or course (Q1)

7 The levels and points-based mechanism (Q3)

8 The teams-based mechanism (Q1, Q3)

9 The gamification approach (Q3)

10 Real use case of the iWarn tool (Q2)

Figure 4 shows an example of the bulletin board entry for the first topic in Table 1. The figure is tagged as the blog is in Spanish. Each topic included a video showing how the system works for that specific functionality, a textual description, and a set of questions that the participants could use to build their argumentation on the proposed solution's utility, challenges, and limitations. The example in Figure 4 includes an introductory video of the architecture and main functionalities of iWarn, and three questions to raise discussion among participants: whether they liked the idea, whether they will use iWarn and which negative and positive aspects they found in participatory early warning.

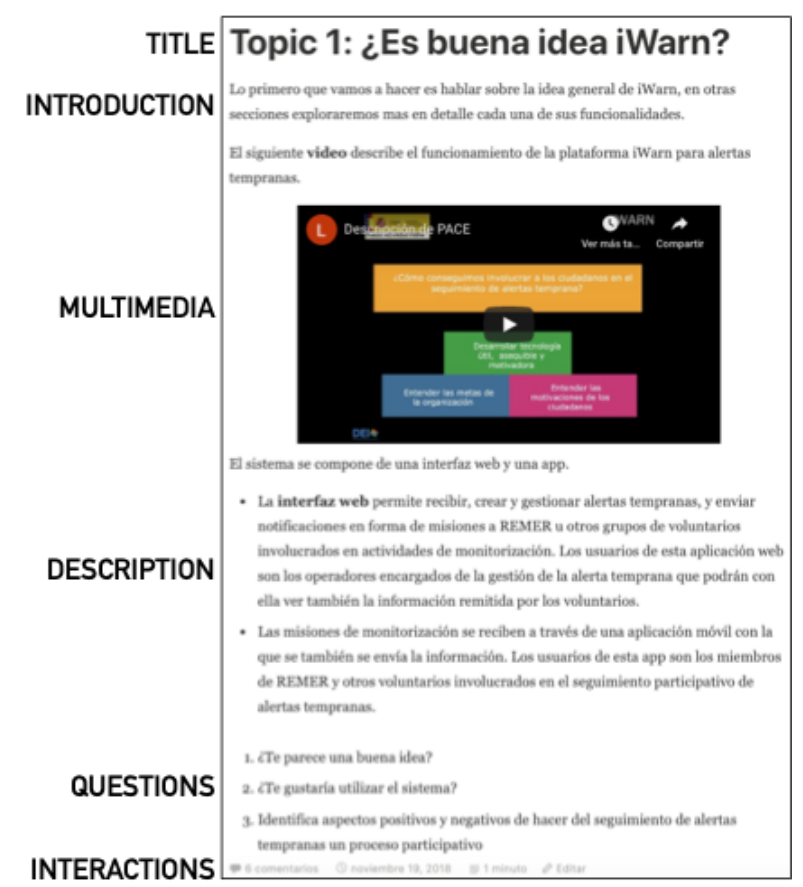

Figure 4. Entry from the EFG blog

To analyze all the answers, we followed a methodology based on grounded theory [24]. The posted comments were categorized using triangulation into three main arguments (communication COM, early warning management EWM, and citizen participation $\mathrm{CP})$ and then they have been coded depending on the 
type of contribution (objectives OBJ, outcomes OUT, causes $\mathrm{CAU}$, consequences $\mathrm{CON}$, and strategy \& actions STR). These codes are adapted from the axial coding paradigm by Strauss and Corbin [25]. Objectives and the causes represent the causal conditions that generate the phenomenon to analyze (i.e., the utility of iWarn). The consequences and the outcomes are the negative and positive characteristics. The strategy $\&$ actions collects proposals for an efficient use of iWarn. We discuss these results using the three main arguments.

Table 2. Main results from the codification

\begin{tabular}{|c|c|}
\hline Args & Results \\
\hline \multirow{8}{*}{ COM } & OBJ. Quick way to track information \\
\hline & OBJ. Widespread audience \\
\hline & CAU. Drop communications \\
\hline & CON. Information overload \\
\hline & STR. Bidirectional communication \\
\hline & STR. Communication among agencies \\
\hline & STR. Data protection \& privacy \\
\hline & STR. Integration with existing channels \\
\hline \multirow{7}{*}{ EWM } & OBJ. Supporting the decision making \\
\hline & OUT. Geo-localized information \\
\hline & CON. Wrong warnings categorization \\
\hline & STR. Integration of data from different sources \\
\hline & STR. Scalable and adaptive system \\
\hline & STR. Threshold for citizen participation \\
\hline & $\begin{array}{l}\text { STR. Categorizing warnings by geographical } \\
\text { areas, types, and sources }\end{array}$ \\
\hline \multirow{24}{*}{$\mathrm{CP}$} & OBJ. Promote a meaningful participation \\
\hline & OBJ. Citizen as human sensor \\
\hline & OBJ. Quick way to receive information \\
\hline & OUT. Segmented information \\
\hline & OUT. Large volume of information \\
\hline & CAU. Continuous involvement in missions \\
\hline & CAU. Overstimulation \\
\hline & $\begin{array}{l}\text { CAU. Lack of trust, subjectivity, lack of } \\
\text { experience and knowledge }\end{array}$ \\
\hline & CON. Missions overload \\
\hline & CON. Tiredness and boredom \\
\hline & CON. Sending not useful information \\
\hline & CON. Risky situation for the citizen \\
\hline & CON. Not getting in touch with the agency \\
\hline & STR. Usable and accessible interfaces \\
\hline & STR. Feedbacks to the citizens \\
\hline & $\begin{array}{l}\text { STR. Be aware of the app goal, scope, and the } \\
\text { privacy issues }\end{array}$ \\
\hline & STR. Public citizens' roles \\
\hline & STR. Automatic information categorization \\
\hline & STR. Allow both open answers and guided \\
\hline & information collection \\
\hline & STR. Trust-based roles \\
\hline & $\begin{array}{l}\text { STR. Points as measure for useful information } \\
\text { and received training courses }\end{array}$ \\
\hline & STR. Change in the team organization \\
\hline & STR. Collaboration \\
\hline
\end{tabular}

Communication. When an early warning is created, the emergency operators in charge can select the communication channel to send the information, including the iWarn mobile app, emails, SMS, social network messages, and radio transmissions. Participants valued iWarn as a way to reach a wider audience. The operators can also directly contact the author of a notification if they want to know more about it. Their main concerns were about privacy and information overload that is discussed again in the next argument.

Citizen Participation. As we can observe from the answers collected during the focus group (see CP section in Table 2), the participation of the citizens in the early warning activities is considered crucial to speed up the bidirectional communication with the authorities. People can act as human sensors in charge of quickly receive and send meaningful information about the risks around them. In an additional study with the first version of the gamified app we validated that gamification can contribute to improve participation as well as civic engagement [26]. However, using the gamified app generated some concerns on experts based on previous experiences interacting with citizens. They worried mainly about the hyper engagement of the citizens for upgrading levels in the gamified app, which could lead to information overload on the emergency operators' side and risky situations on the citizens' side. A strategy to solve this problem could be to automatically retrieve as much data as possible about the context, encourage for team creation, and categorizing the information based on the semantics.

Another weak point is the trustability of data gathered from the citizens that could be solved, for example, assigning roles to people based on their previous experience, knowledge and quality of their participation. In this way, it would be possible to validate received contributions, recognize those thar are really useful and send appropriate feedback to participants. Receiving positive feedback from the authorities can represent another motivation for citizen participation.

Early Warning Management. From the emergency workers' point of view, the most interesting system functionalities are the definition of geographical areas on the map for locating the risks and the citizen participation, and the integration of early warnings coming from external sources, like the National Weather Agency, with the information collected by the citizens. This integration could generate an information overload in particular in case of highly risky situations. An action to take can be to introduce a ranking to establish different categories of sources depending on their level of trust and define thresholds in the early 
warning severity to decide whether to launch a mission for the citizens.

As a result of what we learned from the focus group, the iWarn system was modified. Most changes affect the gamified app though some of them also had an impact in the desktop system. To avoid hyper-engagement some gamification elements are validated by the leader who will be also in charge of motivating participants and filtering information. The final prototype is the one described in section 3 .

\section{Evaluating iWarn EWS}

The last step was the validation of iWarn. Giving the situation created by the COVID19 pandemic experiments with users had more constraints than ever, not only citizens were still under the lockdown but also EM workers were not always available for simulations. At the end we found an opportunity with the Filomena snowstorm to run an experiment to assess the citizens perception of the app. With that purpose we use the concept of user engagement (UE) that focuses on why a specific technology appeals people to use it [27]. UE is was assessed using the UES-Short Form [28] that also included an open question to collect information on the experience with the iWarn application..

\subsection{Scenario of Use}

The evaluation took place right after the snowstorm Filomena that hit several urban areas of Spain that were not used to such storms from the 7th to the 9th of January 2021. The significant amount of snowfall and the low temperatures along with the lack of preparation of citizens and authorities, caused large ice patches during the following week, paralyzing several urban areas, and creating damages that have not been completely arranged ten months later. In this scenario, and once streets were cleared up and the situation was safe, we asked a group of citizens to evaluate the iWarn app by participating in a mission to collect data about potential damages they could identify in their surroundings. At that moment the lock down was still active in the areas affected by the storm, so that citizens could not gather physically. Using the app in a collaborative mission was also an opportunity to engage with other people in a common challenge, so though the activity could not be considered as a sheer early warning it was a good chance to motivate participants to test the app.

\subsection{Experiment}

We recruited twenty-five citizens to participate in the experiment from diverse backgrounds and with different levels of education, between 18 to more of 54 years old (4\% from 18 to $24,8 \%$ from 25 to $34,68 \%$ from 35 to $44,16 \%$ from 45 to $54,4 \%$ from more of 54 years old). Considering that participants' gender may be fluid and impermanent, at the time of the experiment, thirty-two percent of the participants defined themselves as male, sixty percent female, and eight percent nonbinary.

The participants were located mainly in Madrid (n $=21$ participants), Barcelona $(n=2)$, and Valencia $(n=2)$. Participants were organized into five different Filomena missions equitably. Four of the missions took place in Madrid, and one of them between Madrid, Barcelona, and Valencia. One of the researchers played the leader role for all the missions to monitor the users' activity.

Participants received an invitation to join one of the missions on a first-come, first-served basis, except for those who were out of Madrid who were included in the same mission. The duration of the study was three days, after which the missions were closed. Then we contacted each participant to fill the questionnaire.

Once the participant showed their interest in the experiment, they received by email a digital consent form with information about the goals of the study, the voluntary nature of the participation, and the anonymous collection and processing of the data, following the process approved by the ethics committee. We also used their email to access the app and send instructions about how to participate, including safety recommendations.

\subsection{Results}

The participants actively used the app during the three days, sending 106 images $(\mathrm{M}=4.08, \mathrm{SD}=3.5)$ and 91 textual messages $(\mathrm{M}=3.58, \mathrm{SD}=3.13)$. With a participation of $1 / 26$ (lower/highest) total of post per person showing that there has been a significant difference among the individual activity of each participant. The chat was also less used than expected. Both issues suggested that the role of the leader as a facilitator should be encouraged in future exercises.

In the UES-SF questionnaire [28], each question is scored with a 5-point Likert scale (1=strongly disagree, $5=$ strongly agree) and associated to a different category related to the user engagement: Focused Attention (FA), Perceived Usability (PU), Aesthetic Appeal (AA) and Reward (RW). FA measures the feeling of being absorbed in the interaction and losing track of time. AA refers to the attractiveness and visual appeal of the interface. PU is concerned with the usability, the degree 
of control and effort required. RW is related to the overall success of the interaction and the users' willingness to recommend or use the application. For each user, we calculated the UE general score as the average between the categories' scores as suggested in [28]. As the experiment was run in Spanish and we translated the original questionnaire, we first measured the questionnaire's reliability with the Cronbach's Alpha, obtaining a value of 0.84 , where the acceptability threshold is 0.7 by the rule of thumb, and a UE General score average of 3.91 out of 5 and $\mathrm{SD}=0.98$.

Analyzing the results obtained for each of the factors (see Figure 5), the highest values belong to Perceived Usability (PU), with a mean score of 4.40 out of 5 and $\mathrm{SD}=0.97$, followed by Reward (RW) with a mean score of 4.19 out of 5 and $\mathrm{SD}=0.75$. Concerning PU, $92 \%$ of the users positively perceived the interaction with iWarn. This result is also related to the degree of control and the effort invested in using the app. Participants also enjoyed the experience and showed interest in recommending the app and using it in the future, as shown by RW results (84\%). Concerning FA, the result is an average score of 3.43 out of 5 with $\mathrm{SD}=0.99$, meaning that some participants were not totally absorbed in the task during the missions. This issue is related to the fact that the app is used whilst walking on the streets, and in the specific Filomena scenario, people had to keep their attention focused on the ice patches on the ground. This situation has been widely described by the images and text messages sent during the mission. This suggests that personal safety was put ahead of participation. Finally, for AA factor we obtained an average score of 3.46 out of 5 with $\mathrm{SD}=0.76$. This result pointed out some differences among the participants' opinions about the interface and its design.

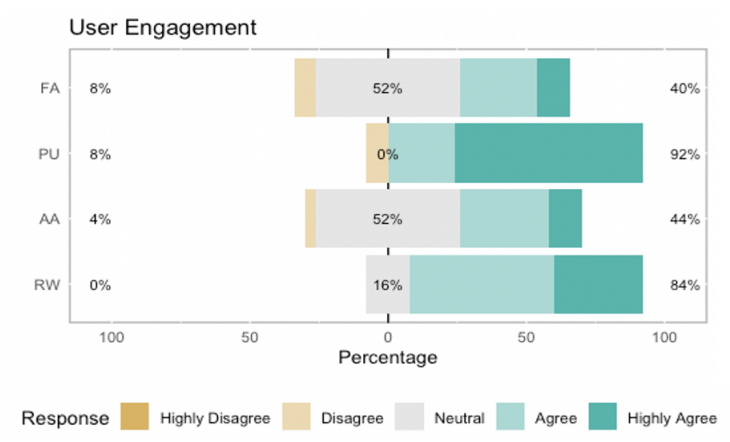

Figure 5. Stacked bar plots for the User Engagement.

Looking at the participants interaction in the chat, we realize that they basically used it to describe the situation where they took the photograph, with comments such as "All this street and crosswalk are cover with ice", "There are a lot of trees in the street which complicates circulation in the neighborhood".
Since the evaluation took part after the Filomena storm most of the photographs are related to different dangers as dangerous tree's branches, accumulation of snow and ice on the roofs and in the streets. Almost all the participants said they would like a more active leader role, which is aligned with one limitation we observed during the experiment, because none of the researchers were trained to manage emergency situations.

From the open question we received significant feedback. The comments are mainly related with two functionalities: the visualization of the participants position in the map, and the possibility to upload photos from the phone gallery in any time and situation. In the first case, some users were not comfortable with the idea of being seen by other participants. As one of them said "It would be interesting to be able to enable/disable your identification and geolocation at certain times". Collaboration was used as a motivator for the participants, but is seems not all them appreciated that option. In any case, full anonymization is not the goal of this app, since all the participants are expected to be part of a team and have to be identified by the operators and the leaders to increase trustability. Anonymous participation can be implemented through social networks or using individual communication mechanisms like the 112 service. The second comment implies being able to upload whatever picture you have in your phone not necessarily geolocated. This kind of functionality will not be valued by decision makers as stated in all the focus groups. Allowing citizens to upload any picture, specially not geolocated, or not being identified will make information useless as trust will be seriously compromised. In general, the majority of participants were positive about using the map with geolocated-photographs, expressing opinions like "It lets you see the problem and connect the picture with the environment" or "It is one of the better ways for the EM services to see that the problem is not a fake".

To sum up, from the results of the experiment it can be assumed that the iWarn mobile application is perceived as a positive technology to improve the collaboration between EM workers and citizens. Moreover, most participants also confirmed their intentions to use the app in the future and recommend it to others in similar situations.

\subsection{Limitations of the experiment}

Integrating and using technologies in EM is always a very challenging process as authorities and emergency workers do not have full availability. The research reported in this paper had to deal with this limitation and with those imposed by the COVID pandemic, which made even more difficult to run experiments since 
neither professionals nor citizens where available for onsite and long term evaluations.

The focus group with experts had to be performed using an asynchronous method based on a well-known technique, the bulletin board, in order to facilitate the participation of geographically scattered experts who would not meet in person easily. This technique has benefits as well as limitations. Whilst it can provoke more reflective contributions since participants do not feel the pressure of being limited to the slot of time devoted to the focus group session, interaction amongst participants might decrease. In any case, there is no way to measure how the opinion of other participants influenced each comment as participants not always quoted other suggestions even though they followed the same thread of discussion. All in all, asynchronous focus groups still provide valuable information as shown in table 2.

Separating the evaluation of the system utility in two groups, experts, and citizens, made it possible to test and improve all its functionalities and improve some issues of the prototype before testing it in a real situation. Given the circumstances it was impossible to execute a complete simulation exercise involving official EM services and citizens but through the two evaluations reported in this paper we collected useful information that can be shared with the research community concerning how citizens and professionals envision this kind of participatory technologies and which design issues have to be considered to integrate participation in a useful, safe and motivating way. Indeed the first prototype evolved from the comments of the focus group to have a more controlled and moderated participation that gives control to professional EM workers and selected volunteers to avoid as much as possible a citizen's hyper motivation that will end up in sending useless information. During the evaluation with citizens, the guiding role of the leader came up as a key feature to engage participants and support interaction and, therefore, the chat became a tool to receive feedback and directions to improve the quality of each individual contribution. More experiments are required to analyze whether this kind of tools can really contribute to improve social capital and make early warning more efficient and sustainable.

\section{Conclusions}

People-centered early warning is expected to improve communities' and individuals' awareness about risks and risk preparation. Moving around to collect data about precursors of potential hazards might make citizens more aware of their environment, vulnerabilities, and the resources they have available. In this paper, we discussed how to integrate citizen- generated information to monitor precursors of potential hazards to get a better picture of the situation by taking profit from mobile and gamified application computing. Mobile computing could support more resilient communities by empowering citizens to participate in this process everywhere and every time. Gamification can act as a motivator to increase participation.

Participatory EM technologies need to take into account the perspective of the other side of the coin. EM workers and decision-makers. The type of participation and information provided by citizens must be carefully co-designed with decision-makers to guarantee that instead of information overload, it supports situation awareness and knowledge generation. For this reason, we opted for an action research approach that integrates different types of stakeholders in the analysis of the problem and the assessment of solutions. Indeed, when evaluated with volunteers who actually work closely with citizens, many issues were raised about how to control their natural enthusiasm to help. This valuable information gathered through an expert focus group helped to ideate a strategy that values both the level of participation and the quality of participation. Thus, the human validation is kept into the system with the team leaders in charge of subjectively evaluating participants' contributions based on their expertise.

We also run a simulation exercise in the real scenario of the Filomena snowstorm. 25 citizens were asked to use the iWarn app for three days and sent images and text messages about potential risks and dangerous situations around them. The results collected show an interest in helping in any hazard situation and improving the efficiency of the EM services which might have an impact into community. Combining the results of the simulation with the information collected from the focus groups with experts helped to adopt design decisions that could support a more participatory yet efficient process.

\section{Acknowledgements}

This work is partly funded by "Comunidad de Madrid en el marco del convenio plurianual con la Universidad Carlos III Madrid en su línea de actuación Excelencia para el Profesorado Universitario-V Plan Regional de Investigación Científica e Innovación Tecnológica 2016-2020" the Spanish Ministry of Economy and Competitiveness Project TIN2016-77690-R “PACE.

\section{References}

[1] de León, J. C. V., Bogardi, J., Dannenmann, S., \& Basher, R. (2006). Early warning systems in the context of disaster risk management. Entwicklung and Ländlicher Raum, 2, 23-25. 
[2] Goldammer, J. G. (1998). Early warning systems for the prediction of an appropriate response to wildfires and related environmental hazards. Health Guidelines for Vegetation Fire Events, 6-9.

[3] de Groot, W. J. et al (2006). Developing a global early warning system for wildland fire. Forest Ecology and Management, 234(1), S10.

[4] Poslad, S., Middleton, S. E., Chaves, F., Tao, R., Necmioglu, O., \& Bügel, U. (2015). A semantic IoT early warning system for natural environment crisis management. IEEE Transactions on Emerging Topics in Computing, 3(2), 246-257.

[5] Alfieri, L., Salamon, P., Pappenberger, F., Wetterhall, F., \& Thielen, J. (2012). Operational early warning systems for water-related hazards in Europe. Environmental Science \& Policy, 21, 35-49.

[6] Herranz, S., Díez, D., Díaz, P., \& Hiltz, S. R. (2012). Exploring the design of technological platforms for virtual communities of practice. In Information Systems for Crisis Response and Management Conference (ISCRAM).

[7] IDNDR - International Decade for Natural Disaster Reduction (1997) Guiding Principles for Effective Early Warning, Communication, IDNDR Secretariat, Geneva, Switzerland

[8] Díaz, P., Carroll, J. M., \& Aedo, I. (2016). Coproduction as an Approach to Technology-Mediated Citizen Participation in Emergency

Management. Future Internet, 8(3), 41.

[9] Endsley MR (2003) Designing for situation awareness: an approach to user-centered design. CRC Press, Boca Raton

[10] Dynes, R. (2006): Social Capital Dealing with Community Emergencies. Homeland Secur. Affairs, 2 , $1-26$.

[11] Boulos, M.N.K.; Resch, B.; Crowley, D.N.; Breslin, J.G.; Sohn, G.; Burtner, R.; Chuang, K.Y.S. (2011): Crowdsourcing, citizen sensing and sensor web technologies for public and environmental health surveillance and crisis management: trends, OGC standards and application examples. Int. J. health geographics, 10(1).

[12] Lanfranchi, V., Wrigley, S. N., Ireson, N., Wehn, U., \& Ciravegna, F. (2014). Citizens' observatories for situation awareness in flooding. In Proc. of 11th International Conference on Information Systems for Crisis Response and Management (pp. 145-154). Sheffield

[13] Norris, F. H., Stevens, S. P., Pfefferbaum, B., Wyche, K. F., \& Pfefferbaum, R. L. (2008). Community resilience as a metaphor, theory, set of capacities, and strategy for disaster readiness. American journal of community psychology, 41(1-2), 127-150.

[14] Waugh, W. L., \& Streib, G. (2006). Collaboration and leadership for effective emergency management. Public administration review, 66(s1), 131-140.

[15] Deterding, S., Dixon, D., Khaled, R., \& Nacke, L. (2011): From game design elements to gamefulness: defining gamification. In Proc. of the 15th Inter.
Academic MindTrek Conference: Envisioning Future Media Environments, pp. 9--15, ACM.

[16] Goodchild, M.F. (2007). Citizens as sensors: the world of volunteered geography. GeoJournal, 69(4), 211-221.

[17] Kanat, I.E., et al. (2013): Gamification of emergency response training: a public health example. International conference on intelligence and security informatics. IEEE.(pp.134.136).

[18] Meesters, K., Ruhe, A., \& Soetanto, M. (2015). Gamification for data gathering in emergency response exercises. In Proc. of the 9th European Conference on Games Based Learning (pp. 672-679).

[19] Herranz, S., Romero-Gómez, R., Díaz, P., \& Onorati, T. (2014). Multi-view visualizations for emergency communities of volunteers. Journal of Visual Languages \& Computing, 25(6), 981-994.

[20] Hevner, A., \& Chatterjee, S. (2010). Design science research in information systems. In Design research in information systems (pp. 9-22). Springer, MA.

[21] Díaz, P., Onorati, T., Romano, M., \& Aedo, I. (2018). Designing Affordable Technologies to Integrate Citizens in Early Warning Activities. In Multidisciplinary Digital Publishing Institute Proceedings 2(19). p. 1253.

[22] Díaz, P., Onorati, T., \& Aedo, I. (2017). A digital knowledge ecosystem to increase participation in emergency warnings and alerts management. In Int. Conference on Green, Pervasive, and Cloud Computing (pp. 700-711). Springer, Cham.

[23] Krueger, R. A. (2014). Focus groups: A practical guide for applied research. Sage publications.

[24] Glaser, B. G., Strauss, A. L., \& Strutzel, E. (1968). The discovery of grounded theory; strategies for qualitative research. Nursing research, 17(4), 364.

[25] Strauss, A., \& Corbin, J. (1990). Basics of qualitative research. Sage publications.

[26] Romano, M, Díaz P., \& Aedo, I. (2021). Gamificationless: may gamification really foster civic participation? A controlled field experiment. J Ambient Intell Human Comput (2021).

[27] O’Brien, H.L. et al. (2018). A practical approach to measuring user engagement with the refined user engagement scale (UES) and new UES short form. Int. Journal of Human Computer Studies. 112, 28-39.

[28] Sekaran, U. 2006. Research Methods for Business. A Skill Building Approach (5th ed.) 